Young people with RMDs, Participation, Doctors and Patients as Partners. Furthermore, in 17 podcasts our member organisations highlighted their activities for people with RMDs. Volunteers vividly described their inside view in the podcasts. In addition we created a historical timeline for the website. Special highlight was a radio programme based on our videos, summarising our activities that was broadcast by 19 radio stations Results: The video clips attracted more viewers than could have been reached with an event in Berlin. For example we generated 35.000 viewers on our YouTube channel by presenting the birthday speech by our president. Altogether we reached successfully approximately 1.5 million people national wide. I will presen the numbers of people our activities reached and feedback we received.

Conclusion: We are convinced going on the right track using digital media as one of our priorities not only in times of pandemic but as a valuable enrichment for all RMD patients on national and international level.

These examples of best practise experiences shall encourage other patient organisations to take on the challenge in pandemic times. By using digital tools and formats patient organisations can succeed in reaching more RMD patients and in staying connected with their members.

Disclosure of Interests: None declared.

DOI: 10.1136/annrheumdis-2021-eular.3063

\section{OP0324-PARE PATIENT INFORMATION IN TIMES OF CRISIS LESSONS LEARNED FROM COVID-19 COMMUNICATION IN RHEUMATOLOGY}

M. M. Bakker ${ }^{1,2}$, T. Luttikhuis ${ }^{1}$, I. Jansen ${ }^{1}$, J. Rademakers ${ }^{2,3}$, M. De Wit ${ }^{4}$, A. Boonen ${ }^{1,2}$, P. Putrik ${ }^{1,2} .{ }^{1}$ Maastricht University Medical Centre, Department of Internal Medicine, Rheumatology Division, Maastricht, Netherlands; ${ }^{2}$ Maastricht University, CAPHRI - Care and Public Health Research Institute, Maastricht, Netherlands; ${ }^{3}$ Nivel - Netherlands Institute for Health Services Research, -Utrecht, Netherlands; ${ }^{4}$ Tools2Use Patient Association, -, Amsterdam, Netherlands

Background: The rapid global spread of COVID-19 required swift action to provide people with rheumatic and musculoskeletal diseases (RMDs) with reliable information. Important issues included the risk of infection and severe illness, (continued) use of medication, temporary closure of clinics, and organization of (semi-) virtual care. People with limited health literacy are a particularly vulnerable group that might have difficulty accessing, understanding, and applying health information.

Objectives: To evaluate (a) key aspects of crisis communication and (b) explicit consideration of people's health literacy needs in communication to people with RMDs during the first wave of COVID-19 in the Netherlands.

Methods: We conducted an explorative qualitative study including seven interviews in May and June 2020 with representatives of organisations (a mixed regional/academic hospital, the association for RMD professionals and two patient organisations) responsible for information provision to people with RMDs in the Netherlands. Interviewees were asked about preparedness (1) and strategy (2) for crisis communication, and content (3) and reach (4) of communication, considering principles of good crisis communication and health literacy. In addition, through systematic screening of websites, social media and emails, we identified and analysed 13 written communications provided to people with RMDs by these organisations during the first three months of the COVID-19 pandemic. We assessed comprehensibility and applicability with the Dutch adapted version of the Patient Education Materials Assessment tool (PEMAT), the outcome being a percentage of adherence to 24 criteria. We assessed difficulty level using an online assessment application (Figure 1), with Common European Framework of Reference for Languages (CEFR) level B1 being the highest acceptable level.

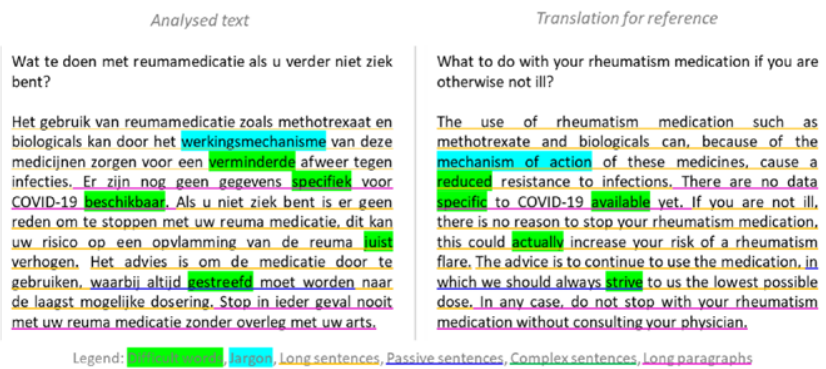

Figure 1. Example of textual assessment using the application. Note: Dutch-language text was used for analysis; the English translation is provided as a reference only and might be of different difficulty.
Results: While admittedly being underprepared, respondents generally perceived their crisis communication as adequate. They quickly adapted to people's needs and changing circumstances and attempted to adapt written and verbal communication to people with limited health literacy. Respondents reported challenges related to the scientific uncertainty, lack of reach, difficulty simplifying information, and being unsure if their communication approach was adequate. Textual assessment showed great variation in applicability (range 60-100\%) and comprehensibility (range $58-100 \%$ ) of these texts, and $69 \%$ of communications were more difficult than B1-level. Considering principles of crisis communication and health literacy, we propose several lessons to be learned for future crises (Table 1).

Table 1. Recommendations for improvement of crisis communication

Preparedness:

Use current experience to establish a future crisis communication plan

Train staff and management in crisis communication and health literate communication Strategy:

Collaborate with relevant organisations to ensure consistency in messages

Inform people early and frequently

Remain transparent about uncertainty

Content:

Adapt information to different people's needs, considering e.g. age, cultural background

Check difficulty level of written information (aim at B1) and adapt accordingly

Ask your audience for feedback

Make sure information is directly applicable in practice

Combat fake news through acknowledgement and counterarguments

Reach:

Use multiple channels

Use diverse outreach strategies to cater to a diverse audience

Overall:

Consider people's health literacy throughout

Conclusion: The rheumatology organisations mostly adhered to principles of crisis communication, and made efforts to adapt information to their audience's needs, including health literacy needs. Nevertheless, important recommendations were drawn which are potentially also relevant for other clinical fields.

Acknowledgements: We thank Mr. Tigran Spaan for providing free access to the online language assessment application.

Disclosure of Interests: None declared.

DOI: 10.1136/annrheumdis-2021-eular.348

\section{LB0001 MAVRILIMUMAB IMPROVES OUTCOMES IN PHASE 2 TRIAL IN NON-MECHANICALLY-VENTILATED PATIENTS WITH SEVERE COVID-19 PNEUMONIA AND SYSTEMIC HYPERINFLAMMATION}

L. Pupim $^{1}$, T. S. Wang ${ }^{2}$, K. Hudock ${ }^{3}$, J. Denson ${ }^{4}$, N. Fourie ${ }^{5}$, L. Hercilla Vasquez ${ }^{6}$, K. Luz ${ }^{7}$, M. Madjid ${ }^{8}$, K. Mcharry ${ }^{9}$, J. F. Saraiva ${ }^{10}$, E. Tobar ${ }^{11}$, T. Zhou ${ }^{1}$, M. Samant ${ }^{1}$, J. Pirrello ${ }^{12}$, F. Fang ${ }^{1}$, J. F. Paolini ${ }^{1}$, A. Pano ${ }^{1}$, B. C. Trapnell ${ }^{3}{ }^{1}$ Kiniksa Pharmaceuticals Corp., Clinical Development, Lexington, United States of America; ${ }^{2}$ University of California, Los Angeles, David Geffen School of Medicine, Los Angeles, United States of America; ${ }^{3}$ University of Cincinnati, Cincinnati Children's Hospital Medical Center, Cincinnati, United States of America; ${ }^{4}$ Tulane University School of Medicine, Critical Care, New Orleans, United States of America; ${ }^{5}$ IATROS International, IATROS International, Bloemfontein, South Africa; ${ }^{6}$ Hospital Nacional Alberto Sabogal Sologuren, Hospital Nacional Alberto Sabogal Sologuren, Callao, Peru; ${ }^{7}$ Rio Grande do Norte Federal University, Rio Grande do Norte Federal University, Rio Grande do Norte, Brazil; ${ }^{8}$ University of Texas Medical School at Houston, McGovern Medical School, Houston, United States of America; ${ }^{9}$ TASK Eden, TASK Eden, George, South Africa; ${ }^{10}$ Instituto de Pesquisa Clínica de Campinas, Instituto de Pesquisa Clínica de Campinas, São Paulo, Brazil; ${ }^{11}$ Hospital Clinico Universidad de Chile, Hospital Clinico Universidad de Chile, Santiago, Chile; ${ }^{12}$ Kiniksa Pharmaceuticals Corp., Business Development, Lexington, United States of America

Background: Granulocyte/macrophage-colony stimulating factor (GM-CSF) is a cytokine both vital to lung homeostasis and important in regulating inflammation and autoimmunity ${ }^{1,2,3}$ that has been implicated in the pathogenesis of respiratory failure and death in patients with severe COVID-19 pneumonia and systemic hyperinflammation. ${ }^{4-6}$ Mavrilimumab is a human anti GM-CSF receptor $\alpha$ monoclonal antibody capable of blocking GM-CSF signaling and downregulating the inflammatory process Objectives: To evaluate the effect of mavrilimumab on clinical outcomes in patients hospitalized with severe COVID-19 pneumonia and systemic hyperinflammation. Methods: This on-going, global, randomized, double-blind, placebo-controlled seamless transition Phase 2/3 trial was designed to evaluate the efficacy and safety of mavrilimumab in adults hospitalized with severe COVID-19 pneumonia and hyperinflammation. The Phase 2 portion comprised two groups: Cohort 1 patients requiring supplemental oxygen therapy without mechanical 